\title{
ESTUDO DE PARÂMETROS CINÉTICOS EM REAÇÕES DE RETICULAÇÃO A PARTIR DE GELATINA
}

\author{
M. T. S. SILVA ${ }^{1}$, J. FERREIRA ${ }^{1}$, D. V. WAY ${ }^{1}$, J. C. PINTO ${ }^{1}$ \\ ${ }^{1}$ Universidade Federal do Rio de Janeiro, Programa de Engenharia Química - \\ PEQ/COPPE \\ e-mail: mairasilva@peq.coppe.urfj.br
}

\begin{abstract}
RESUMO - A utilização de biopolímeros para a produção de filmes surge como mais uma alternativa aos plásticos comerciais, decorrente da sua viabilidade econômica e técnica apresentando um grande potencial de expansão.Estudos prévios mostram que o processo de reticulação de polímeros naturais é muito importante em várias aplicações que requerem a formação de filmes/películas plásticas. Este trabalho teve como objetivo avaliar dados obtidos do acompanhamento cinético da reação de reticulação de um polímero natural, a gelatina. Um mecanismo cinético foi proposto, e por meio deste foi feito uma modelagem matemática a partir do balanço material das espécies envolvidas. Primeiramente foi feita uma avaliação da evolução da massa molar e posteriormente a análise estatística dos dados experimentais, com consequente estimação e avaliação dos parâmetros do modelo proposto por meio do software Matlab. O modelo é validado por meio de dados da massa molar média (Mn) e a massa molar ponderal $(\mathrm{Mw})$. Os dados foram obtidos por meio de variações da concentração de gelatina (Cgel), concentração de glicose (Cgli) e temperatura. A rota poderá constituir, dentro de um conjunto, a base para o desenvolvimento de filmes plásticos para a indústria de alimentos.
\end{abstract}

\section{INTRODUÇÃO}

O desenvolvimento de filmes e coberturas a partir de polímeros naturais tem apresentadoum grande potencial de expansão devido à possibilidade de substituição parcial de materiais plásticos não biodegradáveis.

A gelatina é uma proteína de origem animal, derivada do colágeno, sendo amplamente utilizada no mercado nacional (alimentício) pela facilidade de obtenção e baixo custo. Suas propriedades estão relacionadas principalmente com a composição de aminoácidos, massa molecular média e com o grau de polimerização da cadeia (JOHNSTONBANKS, 1988).Os filmes de proteínas, como a gelatina, apresentam boas barreiras a gases $\left(\mathrm{O}_{2}\right.$ e $\left.\mathrm{CO}_{2}\right)$ somente em ambientes com baixa umidade relativa, em decorrência à 
susceptibilidade do filme em absorver umidade e se dissolver (DAVANÇO et al., 2007).Alguns trabalhos revelam que o maior problema desses filmes está ligado a alta permeabilidade ao vapor de água, devido àsua solubilidade (CARVALHO, 1997).Diante desses, dentre outros aspectos, a busca de meios para solucionar esse problema tem sido um grande desafio para a área de engenharia de polímeros.

Sendo assim, estratégias em busca de solucionar esse problema vêm sendo cada vez mais abordada. A adição de lipídeos em combinação com esses polímeros tem levado a boas propriedades de barreira, mas, por outro lado, propriedades mecânicas indesejáveis.Já a adição de agentes reticulantes é umrecurso muitoutilizado para promover mudanças desejáveis em polímeros sintéticos. Esses agentes tem intuito de interligar as cadeias poliméricaspor meio das reações de reticulação, levando a propriedades finais adequadas, chegando a melhorar permeabilidade ao vapor d'agua.CARVALHO et al. (2005) empregaram dois reticulantes na formação de filmes de gelatina biodegradáveis e observaram que ambos foram capazes de reduzir a solubilidade dos filmes. Além disso, verificaram que as propriedades finais dos filmes podem ser função do arranjo espacial após a reticulação.Esse tratamento,apesar de eficiente, não considera em geral a toxicidade do composto, portanto, seu uso carece de avaliações minuciosas.

Apesar de inúmeros trabalhos relacionados a reticulação de gelatina, a grande maioria dostrabalhos da literatura emprega compostos tóxicos para reticular a gelatina. Além disso, estudos prévios mostram que o processo de reticulação de polímeros naturais é muito pouco compreendido e o desenvolvimento de novos processos são necessários.A reticulação desses materiais é muito importante em várias aplicações que requerem a formação de filmes e películas plásticas biodegradáveis e insolúveis ou resistentes à água, como na indústria de alimentos.Não existe uma rota que explique esse processo e diante disso, o objetivo desse trabalho é analisar os dados estatisticamente em busca da influência das variáveis na evolução das massas,propor modelo rotacional eestudar o comportamento cinético por meio da estimação de parâmetros.

\section{METODOLOGIA}

\subsection{Materiais}

Foram utilizados para o estudo gelatina grau farmacêutico PhEur Gelatin (SigmaAldrich, Alemanha). Como reticulante D-(+)-glicose Anidra (Sigma-Aldrich, França). Outros reagentes foram utilizados como: óleo de girassol, SPAN 80, água destilada para o preparo das reações.

\subsection{Caracterização}

Cromatografia de Permeação em Gel (GPC): A técnica de GPC foi empregada para determinação das massas molares das gelatinas. Tanto reagentes como produtos foram 
caracterizados, de forma a possibilitar o acompanhamento da evolução das massas molares de acordo com as reações realizadas.

\subsection{Estimação de parâmetros}

O mecanismo cinético proposto pode ser na equação (1):

$D_{i}+D_{j} \stackrel{k}{\rightarrow} D_{i+j}(1)$

Para a estimação dos parâmetros cinéticos da reação foi realizado o balançomaterial das espécies envolvidas(gelatina), e aplicando a técnica dos momentosfoi obtidoo seguinte sistema de equações: (equação (2)).

$$
\left\{\begin{array}{c}
\frac{d \lambda_{0}}{d t}=-\frac{1}{2} \frac{k}{V} \lambda_{0}^{2} \\
\frac{d \lambda_{1}}{d t}=0 \\
\frac{d \lambda_{2}}{d t}=\frac{k}{V} \lambda_{1}^{2}
\end{array}\right.
$$

Com $\lambda_{0}$ sendo números total moles, $\lambda_{1} \mathrm{o}$ número total de meros nas cadeia, $\lambda_{2}$ não têm interpretação física, mas representam a heterogeneidade de distribuição de massas molares, $k$ é constante cinética da reação, t o tempo e $V$ o volume de reação.

Portanto, a partir dos balanços é possível deduzir que $\lambda_{1}$ é constante ao longo do tempo, e reduzir o sistema nas seguintes equações:

$$
\begin{aligned}
& \frac{1}{\lambda_{0}}=\frac{1}{2} \frac{k}{V} t-\frac{1}{\lambda_{0_{o}}}(3) \\
& \lambda_{2}=\frac{k}{V} \lambda_{1}^{2} t+\lambda_{2_{o}}(4)
\end{aligned}
$$

Onde $\lambda_{0 o}$ e $\lambda_{2 o}$ são representados pelos valores iniciais de massa molar média (Mn) e massa molar ponderal $(\mathrm{Mw})$, respectivamente.

$$
\operatorname{Com} k=k_{o} e^{-E a / R T}, \lambda_{0}=\frac{1}{M n}, \lambda_{1}=1, \lambda_{2}=M_{W}
$$

é obtido: 


$$
\left\{\begin{array}{l}
M n=\frac{1}{2} \frac{k_{o} e^{-E a / R T}}{V} t+M n_{o} \\
M w=\frac{k_{o} e^{-E a / R T}}{V} t+M w_{o}
\end{array}\right.
$$

Os parâmetros a serem estimados foram $k_{o}$ e $E_{a}$.

Com os resultados obtidos foram criadas 4 réplicas para cada tempo, no programa Excel, com um desvio de $10 \%$. A estimação foi realizada por mínimos quadrados ponderados e implementada por meio do programa Matlab. Foi utilizado um método híbrido para a busca do mínimo das diferenças entre a curva estimada e os dados; primeiramente foi realizado uma busca não determinística com o algoritmo Swarm, e posteriormente uma busca determinística por meio do algoritmo interior-point já implementado no Matlab, sendo o ponto de shootingo ponto achado com a busca no Swarm.

Na Tabela 1 estão dispostos os valores das condições experimentais, fixados em Cglic $(1 \%$ à $4 \%)$, temperatura $\left(50^{\circ} \mathrm{C}, 60^{\circ} \mathrm{C}\right), \mathrm{Cgel}(3 \mathrm{~g}$ e $4,5 \mathrm{~g}) . \mathrm{O}$ tempo de cada reação foi de 30 minutos. As respostas fornecidas para a estimação foram os valores da massa molar ponderal (Mw) e massa molar média (Mn), obtidas por meio das análises de GPC.

Tabela 1 - Tabela de condições experimentais

\begin{tabular}{|c|c|c|c|c|c|c|c|}
\hline \multirow{2}{*}{ Reação } & \multirow{2}{*}{$\begin{array}{l}\text { Água } \\
\text { (g) }\end{array}$} & \multirow{2}{*}{$\begin{array}{l}\text { Óleo } \\
(\mathrm{g})\end{array}$} & \multicolumn{2}{|c|}{ Gelatina } & \multirow{2}{*}{ SPAN $80(\mathrm{~g})$} & \multirow{2}{*}{ Glicose (\%) } & \multirow{2}{*}{ Temperatura $\left({ }^{\circ} \mathrm{C}\right)$} \\
\hline & & & (g) & Tipo & & & \\
\hline 1 & 30 & 120 & 3 & PhEur & 6 & 1 & 50 \\
\hline 2 & 30 & 120 & 3 & PhEur & 6 & 2 & 50 \\
\hline 3 & 30 & 120 & 3 & PhEur & 6 & 3 & 50 \\
\hline 4 & 30 & 120 & 3 & PhEur & 6 & 4 & 50 \\
\hline 5 & 30 & 120 & 3 & PhEur & 6 & 4 & 60 \\
\hline 6 & 30 & 120 & 4,5 & PhEur & 6 & 4 & 50 \\
\hline 7 & 30 & 120 & 4,5 & PhEur & 6 & 4 & 60 \\
\hline
\end{tabular}

\subsection{Análise estatística}

Os dados obtidos foram submetidos a testes estatísticos como ANOVA para avaliar a influência das variáveis na evolução das massas molares. Além disso, foram feitos os testes $t$ student e chi-quadradopara análise de dados experimentais, pois permite estabelecer os limites precisos sobre a região de confiança destes. 


\section{RESULTADOS E DISCUSSÃO}

\subsection{Estimação dos parâmetros cinéticos}

$\mathrm{Na}$ etapa de estimação dos parâmetros foi encontrado o valor da função objetivo de $3,4 \times 10^{11}$, com um tempo de corrida de 2,97 segundos. Assim, por meio do cálculo do mínimo e máximo aceitável estatisticamente para o valor da função, foi encontrado que a faixa de valores está entre 29,95 - 67,82. Portanto, o modelo proposto não corresponde com os dados amostrados.

\subsection{Análise da evolução das massas molares}

Uma vez reticuladas, as moléculas de gelatina têm sua massa molar bastante elevada, o que pode ser facilmente detectado com auxílio da cromatografia de permeação em gel.Apesar de ser uma técnica muito simples e largamente empregada na área depolímeros, não há relatos na literatura de tentativas de acompanhar a cinética dereticulação da gelatina por meio de análises de GPC. Portanto, o grupo tem realizado, aparentemente, os primeiros trabalhos.

Como pode ser observado na Figura 1, contrariamente ao que era esperado, os resultados apresentados não parecem indicar a existência de qualquer aumento apreciável de massa molar ao longo do processamento utilizando glicose como reticulante,o que pode indicar que os material utilizado não foi eficaz parareticular a gelatina, ao contrário do que se afirma na literatura (DIGENIS et al., 1994; CORTESI et al.,1998; WU et al., 2006). Esse comportamento foi observado para todos os experimentos realizados independente das condições empregadas.

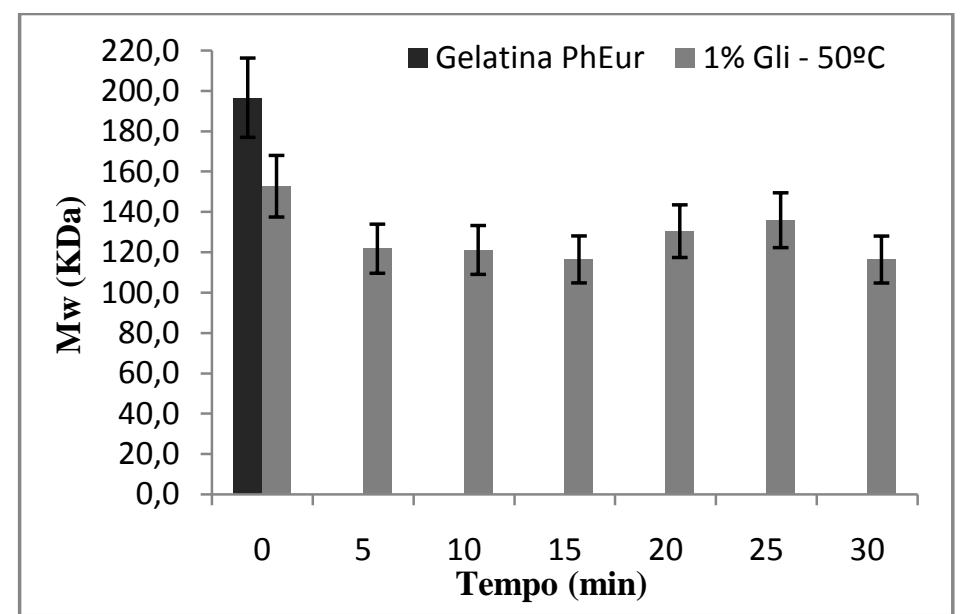

Figura 1- Gráfico da evolução da massa molar ponderal com o tempo para a reação 1, com $1 \%$ de glicose e na temperatura de $50^{\circ} \mathrm{C}$.

No tempo zero são apresentados dois resultados, no qual há uma brusca redução na massa molar da gelatina já nos instantes iniciais da reação.Esse resultado indica a existência de efeitos relevantes sobre a gelatina durante o processo de dissolução, que antes de ser 
adicionada ao óleo deve ser solubilizada a $60^{\circ} \mathrm{C}$. Quando o colágeno passa pelo processo de condicionamento, sua estrutura de tripla hélice característica é apenas parcialmente quebrada (YANNAS, 1972).A queda na massa molar observada pode ser, portanto, uma indicação da continuação desseprocesso de quebra da estrutura, iniciado quando a gelatina começa a ser solubilizada. Além disso, vale ressaltar que a exposição da gelatina a temperatura de $60^{\circ} \mathrm{C}$ pode dar início ao processo de degradação, o que pode explicar também, ao menos em parte, a queda na massa molar observada. No entanto, essa hipótese é pouco provável, porque o processo de redução da massa molar não é continuado ao longo do tempo.

Outro fator que merece destaque é que após a queda inicial no $\mathrm{Mw}$, quando se consideram que o erro da análise de GPC varia em até 10\%, observa-se que a massa molar do material permanece essencialmente constante até o término da reação. Dessa forma, como pode ser observada por meio Figura 2, as massas molares finais das reações com diferentes condições experimentais e concentrações de agente reticulante são estatisticamente similares.

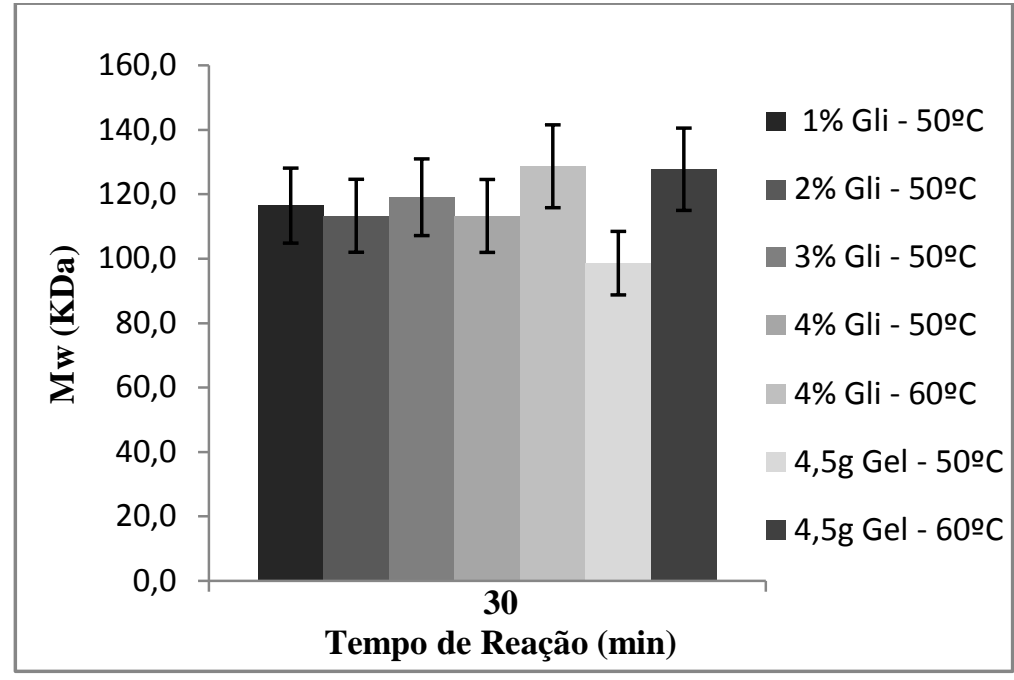

\subsection{Tratamentoestatístico dos dados}

O conhecimento da massa molar dos polímeros como visto anteriormente, é indispensável para possibilitar correta compreensão das propriedades finais. Além disso, o acompanhamento da evolução das massas molares em função da influência das variáveis pode fornecer informações importantes acerca da elaboração de novos processos.

A fim de avaliar a influência da temperatura e da concentração de gelatina na variação da massa molar foi realizada a análise de variância (ANOVA). Para isso, as médias e variâncias reais foram calculadas pelas equações (6) e (7) (PINTO e SCHWAAB, 2007) respectivamente:

$$
t=\frac{\bar{X}_{i}-\mu_{i}}{\frac{s_{i}}{\sqrt{N}}}(6)
$$




$$
\chi_{i}^{2}=(N-1) \frac{s_{i}^{2}}{\sigma_{i}^{2}}
$$

Os dados foram divididos em 2 blocos como pode ser visto na Tabela 2. No primeiro, foi fixada a temperatura e no segundo a concentração de gelatina, afim de facilitar as comparações das grandezas amostrais e assim decidir se as medidas são distintas ou não com grau de confiança de $95 \%$.

Tabela 2 - Média, variância e desvio padrão amostrais, em 2 blocos (temperatura e concentração de gelatina) e seus respectivos intervalos de médias e variâncias reais.

\begin{tabular}{ccccc}
\hline & \multicolumn{2}{c}{ Temperatura $(\mathbf{B 1})$} & \multicolumn{2}{c}{ Concentração de gelatina (B2) } \\
\hline Bloco & $\mathbf{5 0}{ }^{\mathbf{}} \mathbf{C}$ & $\mathbf{6 0}^{\mathbf{}} \mathbf{C}$ & $\mathbf{4 g}$ & $\mathbf{3 g}$ \\
\hline Média & 112,065 & 128,096 & 113,062 & 118,079 \\
\hline Variância & 50,222 & 0,287 & 210,341 & 32,437 \\
\hline Desvio padrão & 7,086 & 0,536 & 14,503 & 5,695 \\
\hline $\boldsymbol{\mu}$ & $109,139<\boldsymbol{\mu}_{1}<114,990$ & $127,71<\boldsymbol{\mu}_{2}<128,48$ & $115,728<\boldsymbol{\mu}_{1}<0,430$ & $102,687<\boldsymbol{\mu}_{2}<123,43$ \\
\hline $\boldsymbol{\sigma}^{\mathbf{2}}$ & $30,620<\boldsymbol{\sigma}^{2}{ }_{1}<97,195$ & $0,136<\boldsymbol{\sigma}^{2}{ }^{2}<1,787$ & $19,777<\boldsymbol{\sigma}^{2}{ }_{1}<62,776$ & $99,515<\boldsymbol{\sigma}^{2}{ }_{2}<701,035$ \\
\hline
\end{tabular}

Os dados resultantes dos testes mostram que tanto as médias como as variâncias não se interceptam para a variável temperatura, ou seja, pode inferir que são diferentes e dessa forma a temperatura pode ter influência nos dados de massa molar. O teste F de Fischer confirma a partirda comparação das variâncias as diferenças entre elas(PINTO e SCHWAAB, 2007),por meio da equação (8):

$$
F=\frac{\overline{S_{1}^{2}}}{\overline{S^{2}}}
$$

No que diz respeito aos resultados referentes à concentração as médias se interceptaram, sendo consideradas estatisticamente não diferentes, mas as variâncias foram diferentes, sendo confirmado pelo teste $\mathrm{F}$ de Fischer que realmente a concentração também podem influenciar na evolução das massas molares em reações de reticulação.

\section{CONCLUSÕES}

Pode-se concluir primeiramente que o modelo cinético proposto não conseguiu representar os dados obtidos experimentalmente, sendo necessária uma análise mais sucinta dos dados, ou ate mesmo a mudança das condições reacionais junto a busca por novos mecanismos com adição de outros componentes. Assim, pode-se notar que o modelo foi proposto baseado no fato de que haveria um aumento das massas com o tempo de reação, o que de fato não ocorreu, assim, não pode-se afirmar que o mecanismo cinético não seja adequado. 
Além disso, pode-se dizer que glicose não se mostrou um bom reticulante para reações com gelatina, fato este observado por meio de dados de massa molar mostraram comportamento estatisticamente similar para as diversas condições empregadas. Testes estatísticos mostram que tanto a temperatura quanto concentração pode influenciar nos valores de massa molar, o que de certa forma pode ser um passo para aprimorar novas condições a serem empregadas para os processos posteriores.

\section{REFERÊNCIAS}

CARVALHO, R. A. "Desenvolvimento e caracterização de biofilmes a base de gelatina". Dissertação de Mestrado. Faculdade de Engenharia de Alimentos. UNICAMP. Campinas, p 42, 1997.

CORTESI, R., NASTRUZZI, C., DAVIS, S.S., 1998, "Sugar Cross-linked Gelatin for Controlled Release: Microspheres and Disks", Biomaterials, v. 19, pp. 1641-1649.

DAVANÇO, T.; TANADA-PALMU, P.; GROSSO, C. "Filmes compostos de gelatina, triacetina, ácido esteárico ou capróico: efeito dopH e da adição de surfactantes sobre a funcionalidade dos filmes". CiênciaeTecnologiade Alimentos., Campinas, 27(2): 408-416, abr.-jun. 2007

DIGENIS, A., GOLD, T.B., SHAH, V.P. "Crosslinking of Gelatin Capsules and its Relevance to their In Vitro/In Vivo Performance", Journal of Pharmaceutical Sciences, v.83, no 7, pp. 915-921, 1994.

JOHNSTON-BANKS, F. A. "Gelatin.” In P. Harris. Food gels. London: Elsevier Applied Science Publishers. pp. 233 e 289, 1990.

PINTO, J.C; SCHWAAB,M. “Análise de Dados Experimentais I Fundamentos de Estatística e Estimação de Parâmetros”. Editora E-papers, 461p, 2007.

WU, H., ZHANG, Z., WU, D., ZHAO, H., YU, K., HOU, Z.“PreparationandDrug Release CharacteristicsofPingyanmycin- loadedDextran Cross-linkedGelatinMicrospheres for EmbolizationTherapy", JournalofBiomedicalMaterialsResearch. Part B: AppliedPolymers, v.78, pp. 56-62, 2006. 